\title{
Language Representation Following Left MCA Stroke in Children and Adults: An fMRI Study
}

\author{
Robyn Westmacott, Mary Pat McAndrews, Gabrielle deVeber
}

\begin{abstract}
Background: In this case series, functional magnetic resonance imaging was used to examine brain networks that mediate different aspects of language function in 4 young adults (17-22 years) with a history of left middle cerebral artery (MCA) stroke in childhood ( $<7$ years of age but after the neonatal period), and five older individuals (42-57 years) with left MCA stroke as adults ( $>40$ years of age). Although it is widely believed that altered lateralization patterns are more likely to occur following early brain injuries compared with later brain injuries, the presumed plasticity of the young brain has been challenged in recent years, particularly in the domain of language. Methods: We explored this issue by contrasting the brain activation patterns of individuals with childhood left MCA stroke and adult left MCA stroke while performing two language tasks: verb generation and picture-word matching. Importantly, both groups showed significant recovery of language function, based on standard clinical indicators. Results: Controls showed left lateralized activation for both tasks, although much more pronounced for verb generation. Adult stroke patients also showed left lateralization for both tasks, though somewhat weaker than controls. Childhood stroke patients exhibited significantly weaker lateralization than the adult group for verb generation, but there was no significant group difference for picture-word matching. Conclusions: These preliminary findings suggest that successful reorganization of language function is more likely to involve bilateral recruitment following left MCA stroke in childhood than in adulthood. Of importance, although childhood stroke patients had primarily subcortical lesions, there were substantial alterations in cortical activation patterns.
\end{abstract}

RÉSUMÉ: Représentation du langage suite à un accident vasculaire cérébral chez l'enfant et chez l'adulte. Contexte: Nous avons utilisé l'imagerie par résonance magnétique pour examiner les réseaux cérébraux qui médient différents aspects de la fonction du langage chez 4 jeunes adultes âgés de 17 à 22 ans atteints d'un accident vasculaire cérébral (AVC) impliquant l'artère cérébrale moyenne (ACM) dans l'enfance (après la période néonatale mais en bas de 7 ans) et chez 5 adultes âgés de 42 à 57 ans dont l'AVC au niveau de l'ACM était survenu à l'âge adulte, alors qu'ils étaient âgés de plus de 40 ans. Bien qu'on estime généralement qu'une altération des patterns de latéralisation soit davantage susceptible de survenir suite à des lésions précoces du cerveau par rapport à des lésions plus tardives, la plasticité présumée du cerveau jeune a été remise en question au cours des dernières années, particulièrement en ce qui concerne le langage. Méthodologie: Nous avons exploré cette question en comparant les patterns d'activation du cerveau au moment où des individus ayant subi un AVC de l'ACM gauche dans l'enfance et des individus ayant subi un AVC de l'ACM gauche à l'âge adulte effectuaient deux tâches langagières : la formation de verbes et l'appariement d'images et de mots. Il est à noter que les deux groupes de sujets présentaient une récupération importante de la fonction du langage, selon les indicateurs cliniques standards. Résultats: Les sujets témoins présentaient une activation latéralisée à gauche lors des deux tâches, bien qu'elle ait été beaucoup plus prononcée lors de la formation de verbes. Les patients adultes atteints d'un AVC présentaient également une latéralisation gauche lors de l'exécution des deux tâches, bien qu'elle ait été plus faible que celle des sujets témoins. Les patients qui avaient subi un AVC dans l'enfance présentaient une latéralisation significativement plus faible que celle du groupe adulte lors de la formation de verbes, mais il n'y avait pas de différence significative entre les groupes lors de l'appariement d'images et de mots. Conclusions: Selon ces constatations préliminaires, une réorganisation efficace de la fonction du langage est plus susceptible d'impliquer un recrutement bilatéral suite à un AVC de l'ACM gauche survenant dans l'enfance qu'à l'âge adulte. Il est important de noter que, bien que les patients qui avaient subi un AVC dans l'enfance présentaient principalement des lésions sous-corticales, ils avaient des altérations importantes des patterns d'activation corticaux.

Keywords: pediatric stroke, MCA stroke, language recovery, fMRI, laterality, language reorganization

doi:10.1017/cjn.2017.44

Can J Neurol Sci. 2017; 44: 483-497

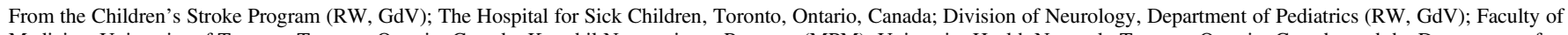
Medicine, University of Toronto, Toronto, Ontario, Canada; Krembil Neuroscience Program (MPM); University Health Network, Toronto, Ontario, Canada; and the Department of

Psychology (MPM), University of Toronto, Toronto, Ontario, Canada

Received December 6, 2016. Final Revisions Submitted February 16, 2017. Date of Acceptance February $21,2017$.

Correspondence to: Robyn Westmacott, Department of Psychology, Hospital for Sick Children, 555 University Avenue, Toronto, ON M5G 1X8, Canada. Email: robyn.

westmacott@ sickkids.ca 
Left middle cerebral artery (MCA) stroke is known to result in significant speech and language deficits in adults, whereas infants and young children with left hemisphere lesions rarely develop persistent aphasic disorders. The resilience of speech and language function in the event of early left hemisphere injury (before 5-10 years of age) has been attributed to enhanced plasticity of the young brain. Recovery of language following brain injury is presumably related to reorganization of functional networks whereby intact brain regions assume new functions to compensate for damaged regions. This capacity for reorganization is thought to decrease with age, resulting in better functional outcomes the younger the age at injury. ${ }^{1}$

Several functional neuroimaging studies suggest that, in adults with left MCA stroke, right hemisphere language dominance is sometimes observed in the acute poststroke stage but rarely persists over time in those with good outcome. ${ }^{1-7}$ In contrast, right hemisphere language dominance appears to be much more common in cases of large left MCA lesions caused by perinatal stroke $^{8-14}$ or left hemisphere lesions accompanied by seizure disorder in the first 5 years of life ${ }^{15-18}$ These findings are consistent with the notion of a "critical period" for left hemisphere language specialization ${ }^{19-21}$ and evidence that left hemisphere language dominance begins very early in childhood and continues to strengthen into adolescence. ${ }^{22}$

However, the literature on language reorganization following early brain injury is not entirely consistent with the notion that language function is transferred to the "nondominant" hemisphere. In a large heterogeneous group of children with epilepsy and left hemisphere lesions of various etiologies with onset of epilepsy before 18 years of age, Anderson et $\mathrm{al}^{23}$ found that the majority exhibited left language dominance and that atypical language lateralization was not associated with age at onset or handedness. Liegeois et $\mathrm{al}^{15}$ found that early lesions in classical language areas (ie. Broca's and Wernicke's areas) were not associated with right hemisphere language transfer but, unexpectedly, right hemisphere transfer was observed when damage involved other left hemisphere regions. Furthermore, some studies using cortical stimulation ${ }^{24}$ and intracarotid amobarbital procedure ${ }^{25}$ have also reported perilesional activation and/or recruitment of novel left hemisphere regions in patients with early left hemisphere lesions. Finally, Raja Beharelle et $\mathrm{al}^{26}$ found a positive correlation between left frontal lateralization and language function in individuals with a history of left perinatal stroke, even though the group as a whole showed increased right hemisphere lateralization relative to controls.

Very few studies have contrasted language reorganization following perinatal and later childhood stroke. Ilves et $\mathrm{al}^{14}$ found a right hemisphere shift in language dominance following perinatal stroke, but continued left hemisphere dominance following stroke in later childhood. However, childhood stroke was associated with poorer language outcome than perinatal stroke, making it difficult to determine if differences in language dominance were due to the timing of the stroke or the degree to which language developed normally. Moreover, because participants were tested as children, this study does not provide insight into differences in mature language representation following perinatal or childhood stroke.

A growing body of evidence suggests that higher level language and cognitive deficits may emerge gradually as children with early brain injuries grow older, and that earlier age at lesion is actually associated with poorer long-term outcome. ${ }^{27,28}$ Specifically, although younger children do to tend to recover more quickly from acute-stage language deficits then older children or adults, many go on to develop significant problems with more sophisticated language skills as they get older, such as narrative discourse, written expression, and verbal fluency. ${ }^{29-31}$ Deficits in these more subtle, high-level language abilities can be very disabling for stroke survivors in the workplace, at school, and in social situations. Thus, enhanced plasticity is not necessarily associated with more positive outcome if compensatory mechanisms are ineffective or disrupt the development of higher level skills. ${ }^{32,33}$ For this reason, it is important to obtain long-term language outcome measures when examining the issue of language reorganization following early brain injury.

The goal of this case series study is to expand on the existing research literature by comparing patterns of successful language reorganization following left MCA stroke in early childhood and adulthood. Using verb generation and picture-word matching tasks in the functional magnetic resonance imaging (fMRI) scanner, we studied four young adults with a history of left MCA stroke before age 7 and five older adults with left MCA stroke after the age of 40, all of whom demonstrated good language outcome on standardized tests. Both of these tasks are among the most commonly used fMRI tasks for studying language. ,6,34-36 $^{2,}$ Moreover, because language tasks rely on a different constellation of language-related abilities, use of multiple tasks is important for obtaining a more comprehensive view of language representation. ${ }^{37,38}$ Here, we have chosen one task that has been shown to be strongly left lateralizing (verb generation) and one that has been found to activate more bilaterally (picture-word matching). ${ }^{2,6,35,36}$ Ours is the first fMRI study to directly compare language lateralization following childhood and adult stroke. Based on neuroimaging findings from control children and children with seizure disorders and left hemisphere lesions of mixed etiology, ${ }^{11,15,22,39}$ we expected to find stronger recruitment of bilateral regions in the childhood stroke group compared with the adult stroke group. However, because our participants sustained left hemisphere stroke after some initial language acquisition (after 2 years of age), we expected to see less pronounced right hemisphere language representation than has been reported in children with large perinatal left hemisphere stroke. ${ }^{10,12-14}$

\section{Methods \\ Participants \\ Childhood Stroke}

Participants were recruited from the Children's Stroke Program at The Hospital for Sick Children in Toronto. Inclusion criteria for the pediatric stroke group were: single left MCA infarct sustained after the neonatal period ( 28 days) but before 13 years of age; native English speaker; and current age of at least 17 years. Four young adults (17-21 years old) with a history of left MCA stroke between 2 and 7 years of age met all criteria and participated in the study. Demographic and neurological information is presented in Table 1. All participants sustained lesions of the left basal ganglia and perisylvian cortex was spared in all except subject $\mathrm{C} 3$. This pattern of damage (lesioned basal ganglia and/or thalamus with cortical sparing) is much more common than isolated cortical lesions following MCA infarcts in children and adolescents because of the underlying mechanism of the stroke, which is typically a transient 
Table 1: Patient demographics and clinical profiles

\begin{tabular}{|c|c|c|c|c|c|}
\hline Subject & Sex & Age at study (years) & Age at stroke (years) & Education (years) & Stroke location \\
\hline \multicolumn{6}{|c|}{ Childhood stroke } \\
\hline $\mathrm{C} 1$ & M & 21 & 2 & 13 & L basal ganglia \\
\hline $\mathrm{C} 2$ & $\mathrm{~F}$ & 19 & 5 & 14 & L thalamus \\
\hline $\mathrm{C} 3$ & M & 18 & 5 & 13 & L basal ganglia \\
\hline $\mathrm{C} 4$ & M & 17 & 7 & 12 & L basal ganglia and frontoparietal cortex \\
\hline \multicolumn{6}{|c|}{ Adult stroke } \\
\hline A1 & $\mathrm{F}$ & 45 & 43 & 23 & $\mathrm{~L}$ posterior MCA, temporoparietal infarct \\
\hline A2 & M & 49 & 47 & 12 & $\mathrm{~L}$ anterior MCA, perisylvian infarct \\
\hline A3 & M & 57 & 56 & 14 & $\mathrm{~L}$ anterior MCA, perisylvian infarct \\
\hline A4 & $\mathrm{F}$ & 52 & 50 & 17 & $\mathrm{~L}$ anterior MCA, perisylvian infarct \\
\hline A5 & $\mathrm{F}$ & 43 & 41 & 12 & $\begin{array}{l}\mathrm{L} \text { anterior MCA, } \\
\text { dorsolateral frontal infarct }\end{array}$ \\
\hline
\end{tabular}

$\mathrm{F}=$ female $\mathrm{L}=$ left $\mathrm{M}=$ male.

inflammation of the MCA stem. ${ }^{40-43}$ However, because the young brain typically has better overall perfusion and collateral blood flow than the older brain, children and adolescents are less likely to sustain large cortical infarcts in addition to the subcortical damage. All participants demonstrated aphasic deficits in the acute period but showed substantial recovery and subsequent development of spoken language skills (see the following section for performance on standardized language tests). All participants were native righthanders, though participants $\mathrm{C} 2$ and $\mathrm{C} 3$ switched to left-hand dominance following the stroke. None had global intellectual impairment, epilepsy, congenital heart disease, sickle cell disease, malignancy, recurrent stroke, or other neurological comorbidities.

\section{Adult Stroke}

Participants were recruited from the Stroke Program at Toronto Western Hospital. To be included in the adult stroke group, participants must have sustained a single left MCA infarct sustained after 30 years of age and be a native English speaker. Exclusion criteria were neurological comorbidities, sickle cell disease, moyamoya disease, current seizure disorder, congenital or acquired heart disease, malignancy, bilateral lesions, and stroke recurrence. Five older adults (42-57 years of age) who sustained a left MCA stroke after the age of 40 also participated in the study. All sustained damage to the left perisylvian cortex with subcortical sparing. Participants were all at least 1 year poststroke at the time of the study. All are right-handed native English speakers and experienced aphasic deficits in the acute stroke period. All participants made a good recovery of spoken language skills, as evidenced by standardized test performance (see the following section). Exclusion criteria were recurrent stroke, dementia, psychiatric disturbance, epilepsy, sickle cell disease, cardiac disease, or other neurological disorders. The demographic and neurological profiles of these patients are presented in Table 1.

\section{Controls}

A control group of ten right-handed healthy adults (mean age, 50.4 years; standard deviation, 3.4 years) also participated. All are native English speakers with no neurological or psychiatric history.

\section{Neuropsychological Assessment}

Before the scanning session, patients and controls underwent a brief neuropsychological assessment consisting of the two-subtest version of the Wechsler Abbreviated Scale of Intelligence; the Boston Diagnostic Aphasia Exam, short form; letter and category fluency; the Wechsler Test of Adult Reading; and the Pyramids and Palm Trees Test of semantic memory. Neuropsychological test scores are presented in Table 2.

\section{fMRI Tasks}

\section{Verb Generation}

Participants were asked to silently generate a verb that is associated with a particular concrete noun (e.g. dog - BARKS). The baseline condition involved silent viewing of symbol strings (e.g. $++\$ \# \$$ ), each five items long; subjects were asked to fixate on the stimuli but make no response. Stimuli were presented at the rate of one every 4 seconds in blocks of five, for a total of six blocks. Thus, there were 30 trials for each of the verb generation and baseline conditions. A practice session with ten different stimuli was carried out before the scanning session. After the scanning session, participants were asked to perform the verb generation task again outside of the scanner, with the same stimuli, to ensure that they were performing correctly. This postscanning test session provided an estimate of response accuracy, though we recognize that this introduces the possibility of practice effects.

\section{Picture-Word Matching}

Participants were asked to press the left or right mouse button to indicate which of two words matched a black-and-white line drawing. There were a total of 30 drawings depicting common items from a variety of categories including animals, foods, furniture, vehicles, and tools. In the baseline condition, participants were asked to indicate which symbol string matched the large symbol in the center of the stimulus. Stimuli were presented at the rate of one every 4 seconds in blocks of five, for a total of six blocks (30 trials for each condition). A practice session 
was performed with each subject before entering the scanner. Tasks were run in a standard order for all participants.

This project was approved by Research Ethics Boards at the University Health Network and The Hospital for Sick Children, and all data were collected and handled in accordance with ethical requirements.

\section{Data Analysis}

\section{Image Acquisition}

Images were obtained on a 1.5 T EchoSpeed MRI (GE Medical Systems, Milwaukee, WI). The head of each participant was stabilized in a standard quadrature head coil with a pillow to minimize motion artifact. A mirror was adjusted to ensure that participants could easily see the projection screen on which the stimuli were displayed. A laptop computer with E-PRIME software generated the visual stimuli and recorded response accuracy and reaction time. A magnet-compatible three-buttoned mouse was used to record responses. T1-weighted structural images (120 axial slices; slice thickness, $11.5 \mathrm{~mm}$; field of view, $200 \mathrm{~mm}$ ) followed by T2*-weighted functional images (spiral acquisition) sensitive to blood oxygenation level-dependent contrast were obtained for each participant (25 axial slices; slice thickness, $4.4 \mathrm{~mm}$; repetition time, $2 \mathrm{~s}$; flip angle , $82^{\circ}$; echo time, $40 \mathrm{~ms}$; field of view, $200 \mathrm{~mm}$ ).

\section{Image Analysis}

Functional images were preprocessed and analyzed with the Statistical Parametric Mapping-2 software package. ${ }^{44}$ Images were realigned to correct for motion, corrected for within-frame time of acquisition, spatially normalized, and smoothed with a 7.6-mm full-width half-max Gaussian kernel. Functional maps were overlaid onto T1 anatomical scans. Activation maps were generated by contrasting each task condition with a baseline condition (viewing symbol strings for verb generation, symbol matching for pictureword matching) at a corrected threshold of $p=0.05$. Random-effects analysis was used for the control data. A family-wise error corrected threshold of $\mathrm{p}<0.05$ was used for controls and patients.

One global indicator that $\mathrm{we}^{37}$ and others ${ }^{3,26,45,46}$ have used to describe the relative contribution of left and right hemispheres to language task $\mathrm{I}$ is the Laterality Index, defined as $(\mathrm{L}-\mathrm{R}) /(\mathrm{L}+\mathrm{R})$, where $\mathrm{L}$ represents the number of activated voxels in the left hemisphere and $\mathrm{R}$ represents the number of activated voxels in the right hemisphere. Lateralization indices (LIs) were calculated by group for each task, using the entire supratentorial region for each hemisphere, using voxels that were greater for the task condition than the control condition. Only voxels that exceed threshold (in this case, family-wise error of $\mathrm{p}<0.05$ ) are entered in to the equation. Thus, LIs can range from -1.0 to +1.0 ; by convention, values between -0.2 and +0.2 represent bilateral language distribution; values between -0.2 and -1.0 represent right hemisphere dominance; and values between +0.2 and +1.0 represent left hemisphere dominance. Values between \pm 0.5 and \pm 1.0 are considered to reflect strong hemispheric dominance . $^{3,45,4 \overline{6}}$

\section{RESULTS}

\section{Behavioral Data}

Controls performed at ceiling on both tasks (mean, 99.2\%; standard deviation, 1.15; corrected for both). In the verb generation task, response accuracy was defined as generating an appropriate verb within the allotted time period. In the picture-word matching task, response accuracy was defined as the selection of the correct match. As shown in Table 2, all patients performed above $85 \%$ correct on both tasks, with the exception of adult patient A5 ( $76 \%$ correct on verb generation).

Table 2: Neuropsychological profiles, experimental task performance, and LIs for patients

\begin{tabular}{|c|c|c|c|c|c|c|c|c|c|}
\hline & \multicolumn{4}{|c|}{ Childhood stroke } & \multicolumn{5}{|c|}{ Adult stroke } \\
\hline & C1 & $\mathrm{C2}$ & $\mathbf{C 3}$ & $\mathrm{C4}$ & A1 & A2 & $\mathbf{A 3}$ & A4 & A5 \\
\hline BDAE score $(\%)$ & 95 & 98 & 90 & 98 & 98 & 97 & 95 & 97 & 89 \\
\hline WTAR & 110 & 106 & 104 & 102 & 120 & 102 & 98 & 106 & 94 \\
\hline \multicolumn{10}{|l|}{ WASI-III } \\
\hline Full Scale IQ & 116 & 102 & 98 & 99 & 146 & 96 & 92 & 105 & 90 \\
\hline Verbal IQ & 114 & 100 & 96 & 98 & 138 & 90 & 88 & 100 & 86 \\
\hline Performance IQ & 114 & 105 & 101 & 100 & 126 & 98 & 96 & 108 & 92 \\
\hline \multicolumn{10}{|l|}{ Verbal fluency } \\
\hline Letter cues & 80 & 120 & 95 & 95 & 115 & 80 & 80 & 85 & 70 \\
\hline Category cues & 100 & 115 & 100 & 90 & 110 & 85 & 80 & 95 & 70 \\
\hline Pyramids and Palm Trees, 3 picture version (raw score) & $26 / 26$ & $26 / 26$ & $26 / 26$ & $26 / 26$ & $26 / 26$ & $26 / 26$ & $25 / 26$ & $26 / 26$ & $25 / 26$ \\
\hline Percent correct on verb generation & 100 & 96.7 & 87.8 & 96.7 & 100 & 93.3 & 93.3 & 90.0 & 76.7 \\
\hline LI: verb generation & -0.18 & +0.31 & +0.07 & +0.22 & +0.64 & +0.66 & +0.74 & +0.34 & +0.21 \\
\hline Percent correct on picture-word matching & 96.7 & 100 & 96.7 & 93.3 & 96.7 & 100 & 96.7 & 96.7 & 90.0 \\
\hline LI: picture-word matching & +0.13 & +0.14 & -0.45 & +0.11 & +0.10 & +0.59 & +0.55 & -0.56 & +0.15 \\
\hline
\end{tabular}

Unless otherwise indicated, all psychometric test scores have been translated into standard scores (mean, 100; standard deviation, 15).

$\mathrm{BDAE}=$ Boston Diagnostic Aphasia Examination, short form; WASI $=$ Wechsler Abbreviated Scale of Intelligence; WTAR $=$ Wechsler Test of Adult Reading. 


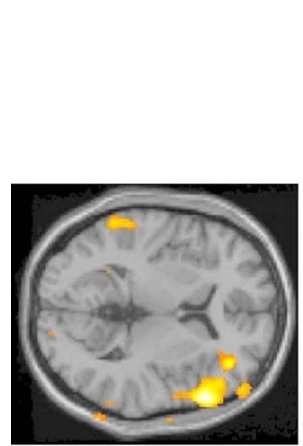

Control $\mathrm{LI}=+\mathbf{0 . 5 8}$

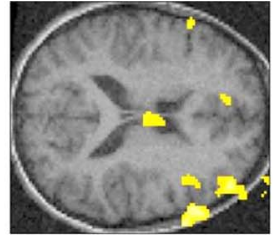

A1 $\mathrm{LI}=+\mathbf{0 . 6 4}$

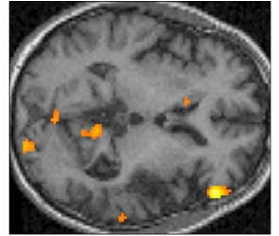

A2 $\mathrm{LI}=+0.66$

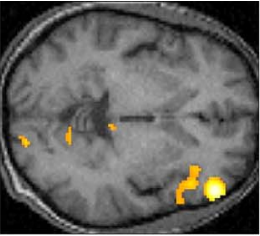

A3 $\mathrm{LI}=+0.74$

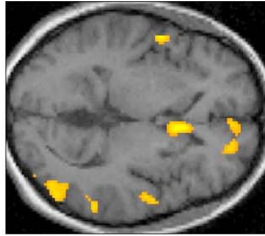

A4 $\mathrm{LI}=+\mathbf{0 . 3 4}$

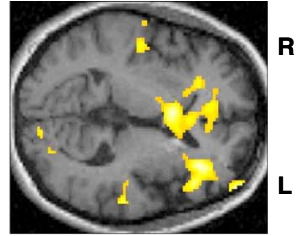

A5 $\mathrm{LI}=+0.21$

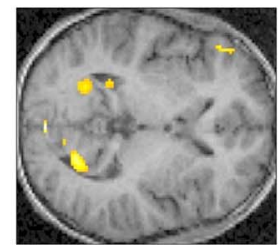

C1 $\mathrm{LI}=\mathbf{- 0 . 1 8}$

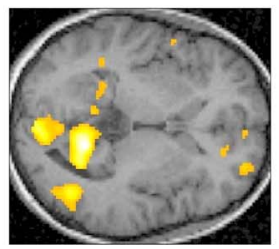

C2 $\mathrm{LI}=+\mathbf{0 . 3 1}$

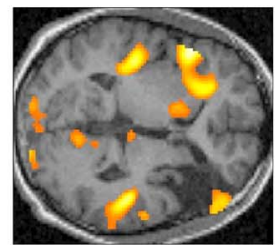

C3 $\quad \mathrm{LI}=+\mathbf{0 . 0 7}$

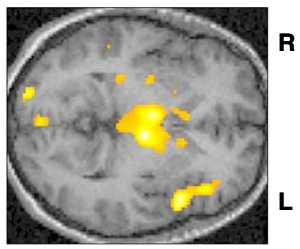

C4 $\mathrm{LI}=+\mathbf{0 . 2 2}$

Figure 1: Activation patterns for verb generation > baseline in controls, adult stroke (A1-A5), and childhood stroke (C1-C4). LIs are presented below each subject's activation map.

\section{LIs}

LIs for controls and the two patient groups are shown in Figures 1 and 2. Controls showed left lateralized activation for both tasks, although much more pronounced for verb generation. The adult stroke patients also showed left lateralization for both tasks, with no statistically significant difference from controls in a non-parametric Mann-Whitney $U$ test (Verb Generation: $\mathrm{t}(13)=-1.92, \mathrm{p}=\mathrm{NS}$; Picture Naming: $\mathrm{t}(13)=-1.35, \mathrm{p}=\mathrm{NS})$. In contrast, the childhood stroke patients exhibited significantly weaker LIs than the adult group for verb generation $(t(7)=2.76$, $\mathrm{p}<0.01$ ), but there was no significant group difference for picture-word matching $(\mathrm{t}(7)=0.687, \mathrm{p}=\mathrm{NS})$.

LIs for individual participants are presented in Table 2 and language maps are shown in Figures 3 and 4. In the adult stroke group, three of the five patients showed strong left lateralization on verb generation $(+0.64,+0.74)$, whereas the other two showed moderate left lateralization $(+0.21,+0.34)$. On picture-word matching, two of the adult stroke patients showed strong left lateralization $(+0.55,+0.59)$, two showed bilateral activation
$(+0.10,+0.15)$ and one showed strong right lateralization $(-0.56)$. In the childhood stroke group, two patients showed moderate left lateralization on verb generation $(+0.22,+0.31)$ and two patients showed bilateral activation $(+0.07,-0.18)$. On picture-word matching, three of the childhood stroke patients showed bilateral activation $(+0.11,+0.13,+0.14)$ and one showed moderate right lateralization $(-0.45)$.

\section{Regions of Significant Activation: Controls}

As Table 3 indicates, control participants performing the verb generation task activated a network of left hemisphere regions including inferior frontal, middle frontal, superior frontal, inferior parietal, superior temporal, and cingulate. Broca's area emerged as the clear point of maximum activation. A few right hemisphere regions were also activated (inferior frontal, superior frontal, superior temporal, cingulate), although to a much lesser extent than their left hemisphere homologues. In the picture-word matching task, controls activated left hemisphere regions including inferior and medial frontal, middle temporal, cingulate, dorsomedial thalamus, and

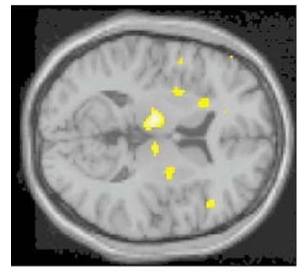

Controls $\mathrm{LI}=+\mathbf{0 . 2 6}$

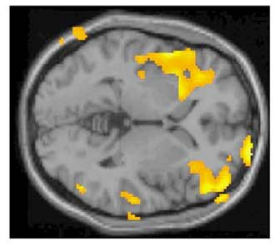

A1 $\mathrm{LI}=+0.10$

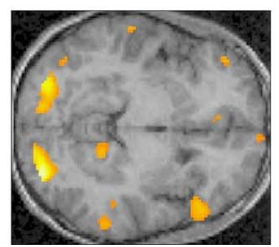

$\mathrm{C} 1 \mathrm{LI}=+\mathbf{0 . 1 3}$

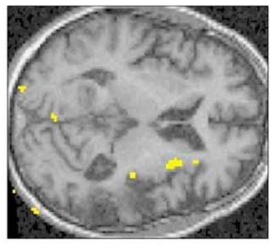

$\mathrm{A} 2 \mathrm{LI}=+0.59$

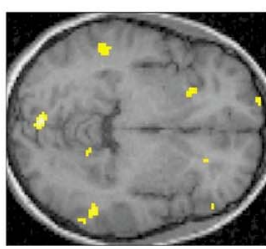

$\mathrm{C} 2 \mathrm{LI}=+\mathbf{0 . 1 4}$

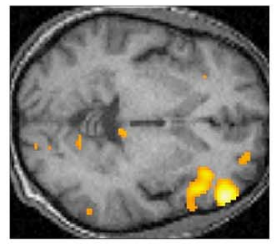

A3 $\mathrm{LI}=+0.55$

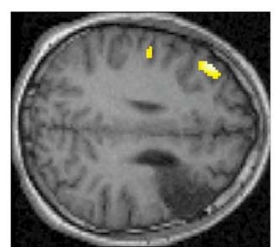

C3 $\mathrm{LI}=-\mathbf{0 . 4 5}$

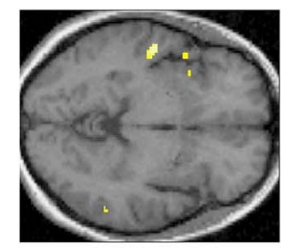

$\mathrm{A} 4 \mathrm{LI}=-0.56$

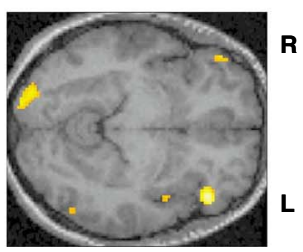

$\mathrm{C} 4 \mathrm{LI}=+\mathbf{0 . 1 1}$

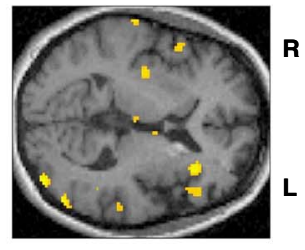

A5 $\mathrm{LI}=+0.15$

Figure 2: Activation patterns for picture-word matching > baseline in controls, adult stroke (A1-A5,) and childhood stroke (C1-C4). LIs are presented below each subject's activation map. 


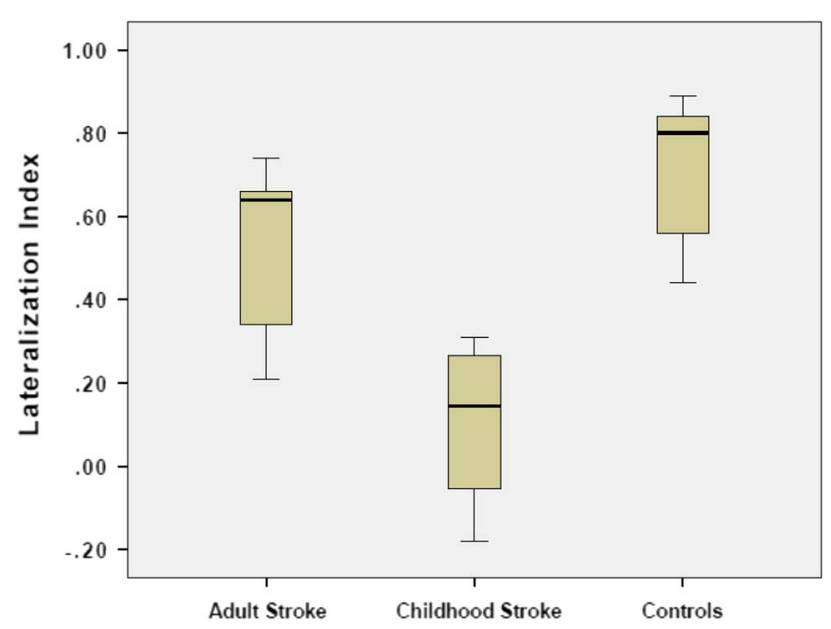

Figure 3: Box plots depicting the variability in LIs for controls, adult stroke (A1-A5), and childhood stroke (C1-C4) on the verb generation task.

parahippocampal gyrus. Maximum activations occurred in left inferior and medial frontal areas, but to a lesser extent than in the verb generation task. Bilateral activation was observed in several regions including superior temporal, cuneus, and fusiform/lingual gyrus.

\section{Regions of Significant Activation: Adult Stroke}

In the verb generation task (Table 4, Fig. 1), all five of the adult stroke patients showed left inferior frontal activation but, unlike controls, this was not the region of maximum activation. Rather, the strongest activations were seen in other regions of left frontal cortex (middle, medial, and superior frontal) and, in some cases, left temporal regions. Of note, only two of the adult stroke patients showed activation of Broca's area (BA 44/45); the other three demonstrated activation other areas within the left inferior and middle frontal gyri (BA 46/9). In contrast to controls, none of the adult stroke patients activated the left angular gyrus and only two patients showed left inferior parietal activation. Moreover, right frontal regions and bilateral occipital/fusiform regions were

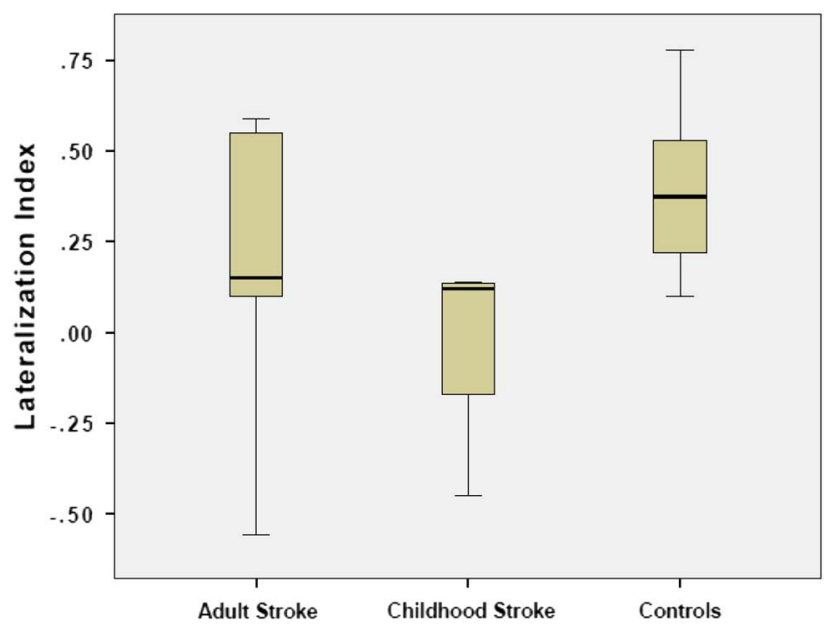

Figure 4: Box plots depicting the variability in LIs for controls, adult stroke (A1-A5), and childhood stroke (C1-C4) on the picture-word matching task.
Table 3: Areas activated by control subjects during verb generation and picture-word matching

\begin{tabular}{|c|c|c|c|}
\hline Region of activation & $\mathbf{B A}$ & $\mathbf{x y} \mathbf{z}$ & t value \\
\hline \multicolumn{4}{|l|}{ Verb generation } \\
\hline \multicolumn{4}{|l|}{ Inferior frontal } \\
\hline Left & 45 & $-55,24,10$ & 15.29 \\
\hline Right & 47 & $30,31,-2$ & 7.25 \\
\hline Middle frontal, left & 46 & $-57,32,23$ & 14.00 \\
\hline \multicolumn{4}{|l|}{ Superior frontal } \\
\hline Left & 8 & $-2.19,48$ & 13.64 \\
\hline Right & 6 & $10,10,49$ & 6.23 \\
\hline Medial frontal, left & 6 & $-6,6,50$ & 11.48 \\
\hline Inferior parietal, left & 40 & $-48,-50,34$ & 7.54 \\
\hline Angular gyrus, left & 39 & $-55,-58,37$ & 7.54 \\
\hline Middle temporal, left & 21 & $-71,-4,-7$ & 10.52 \\
\hline \multicolumn{4}{|l|}{ Superior temporal } \\
\hline Left & 22 & $-71,-40,18$ & 7.03 \\
\hline Right & 22 & $59,-40,8$ & 9.09 \\
\hline Fusiform, left & 37 & $-48,-35,-7$ & 4.53 \\
\hline Cuneus, left & 17 & $-10,-91,2$ & 5.91 \\
\hline \multicolumn{4}{|l|}{ Cingulate } \\
\hline Left & 31 & $-20,-31,40$ & 4.61 \\
\hline Right & 29 & $16,-40,15$ & 6.15 \\
\hline Parahippocampal, left & 19 & $-28,-52,1$ & 4.78 \\
\hline Insula, left & 13 & $-42,-4,-5$ & 4.10 \\
\hline Basal ganglia (putamen), left & $\mathrm{n} / \mathrm{a}$ & $-30,-10,0$ & 4.06 \\
\hline \multicolumn{4}{|l|}{ Picture-word matching } \\
\hline \multicolumn{4}{|l|}{ Inferior frontal } \\
\hline Left & $9 / 44$ & $-57,15,29$ & 6.78 \\
\hline Right & 44 & $63,14,17$ & 3.60 \\
\hline Medial frontal, left & 6 & $-10,-22,61$ & 6.25 \\
\hline Cingulate, left & 31 & $-2,-69,17$ & 6.09 \\
\hline \multicolumn{4}{|l|}{ Inferior parietal } \\
\hline Left & 40 & $-34-4241$ & 4.55 \\
\hline Right & 40 & $44-4646$ & 5.01 \\
\hline Middle temporal, left & 21 & $-67,-52,-6$ & 4.94 \\
\hline \multicolumn{4}{|l|}{ Superior temporal } \\
\hline Left & 22 & $-65,-47,21$ & 4,62 \\
\hline Right & 21 & $65,-12,2$ & 5.34 \\
\hline \multicolumn{4}{|l|}{ Cuneus/precuneus } \\
\hline Left & 19 & $-28,-87,39$ & 5.02 \\
\hline Right & 19 & $24,-87,32$ & 5.04 \\
\hline \multicolumn{4}{|l|}{ Fusiform/inferior temporal } \\
\hline Left & 17 & $-20,-81,3$ & 4.38 \\
\hline Right & 19 & $26,-66,-10$ & 4.86 \\
\hline Dorsomedial thalamus, left & $\mathrm{n} / \mathrm{a}$ & $-10,-15,8$ & 5.33 \\
\hline Parahippocampal gyrus, left & 36 & $-55,39,17$ & 5.29 \\
\hline Basal ganglia (putamen), right & $\mathrm{n} / \mathrm{a}$ & $24-515$ & 5.16 \\
\hline Insula, left & 13 & $-42,-22,-6$ & 4.11 \\
\hline
\end{tabular}

Talairach coordinates $(\mathrm{x}, \mathrm{y}, \mathrm{z})$ are reported. 
Table 4: Areas activated by adult stroke patients during verb generation and picture-word matching

\begin{tabular}{|c|c|c|c|c|c|c|c|c|c|c|}
\hline \multirow[t]{2}{*}{ Region of activation } & \multicolumn{2}{|l|}{ A1 } & \multicolumn{2}{|l|}{ A2 } & \multicolumn{2}{|l|}{$\mathbf{A 3}$} & \multicolumn{2}{|l|}{ A4 } & \multicolumn{2}{|l|}{ A5 } \\
\hline & $\mathbf{x ~ y ~ z ~ ( B A ) ~}$ & t value & $\mathbf{x ~ y ~ z ~}(\mathbf{B A})$ & t value & $\mathbf{x ~ y ~ z ~ ( B A ) ~}$ & t value & $\mathbf{x}$ y z $(\mathbf{B A})$ & $t$ value & $\mathbf{x}$ y z $(\mathbf{B A})$ & t value \\
\hline \multicolumn{11}{|l|}{ Verb generation } \\
\hline \multicolumn{11}{|l|}{ Inferior frontal } \\
\hline Left & $-481819(45)$ & 5.47 & $-53441(46)$ & 7.60 & $-534214(46)$ & 8.67 & $381127(9)$ & 5.91 & $-50924(44)$ & 4.49 \\
\hline Right & $441614(44)$ & 3.80 & 44351 (47) & 3.58 & $652623(45)$ & 5.65 & $3031-6(47)$ & 4.29 & $5515-1(47)$ & 6.32 \\
\hline \multicolumn{11}{|l|}{ Middle frontal } \\
\hline Left & $-464717(10)$ & 6.40 & $-602730(9)$ & 12.01 & $-50519(46)$ & 9.40 & $-521728(9)$ & 10.53 & $-463222(46)$ & 5.19 \\
\hline Right & & & $5352-4(10)$ & 4.01 & $504916(46)$ & 7.74 & $4036-10(47)$ & 4.51 & $424427(9)$ & 6.11 \\
\hline \multicolumn{11}{|l|}{ Superior frontal } \\
\hline Left & $-41761(6)$ & 4.95 & $144434(8)$ & 4.04 & $-26327(10)$ & 6.58 & $-2564(6)$ & 6.62 & $-24585(10)$ & 6.44 \\
\hline Right & $226610(10)$ & 3.73 & & & $164921(9)$ & 5.63 & $1256-6(10)$ & 5.03 & $8747(10)$ & 6.14 \\
\hline \multicolumn{11}{|l|}{ Medial frontal } \\
\hline Left & $-4-1664(6)$ & 3.87 & & & $-22345(8)$ & 10.17 & $-21648(6)$ & 7.62 & $-1-2464(6)$ & 7.15 \\
\hline Right & $124717(9)$ & 4.43 & & & 64037 (6) & 5.95 & $1438-10(10)$ & 5.33 & $18159(6)$ & 7.93 \\
\hline \multicolumn{11}{|l|}{ Inferior parietal } \\
\hline Left & & & & & & & $-40-5442(40)$ & 5.98 & $-48-2932(40)$ & 3.72 \\
\hline Right & & & & & & & $59-3737(40)$ & 3.83 & $53-3342(40)$ & 3.47 \\
\hline \multicolumn{11}{|l|}{ Angular gyrus } \\
\hline \multicolumn{11}{|l|}{ Left } \\
\hline \multicolumn{11}{|l|}{ Right } \\
\hline \multicolumn{11}{|l|}{ Middle temporal } \\
\hline Left & $-71-43-9(21)$ & 6.55 & $-50-418(21)$ & 3.98 & $-57-585(21)$ & 8.60 & $-56-466(21)$ & 9.81 & $-71-37-9(21)$ & 5.59 \\
\hline Right & $69-43-7(21)$ & 3.32 & & & & & $67-37-7(21)$ & 4.03 & $67-16-9(21)$ & 3.89 \\
\hline \multicolumn{11}{|l|}{ Superior temporal } \\
\hline Left & $-65-214(22)$ & 4.50 & $-60-4021(22)$ & 3.98 & $-381-13(38)$ & 5.08 & $-61-4413(22)$ & 5.98 & $-67102(22)$ & 4.24 \\
\hline Right & $6119-7(38)$ & 3.62 & & & & & $67-2416(42)$ & 4.89 & & \\
\hline \multicolumn{11}{|c|}{ Fusiform/inferior temporal } \\
\hline Left & $40-78-14(19)$ & 3.43 & $-20-98-22(18)$ & 3.17 & $-65-59-11(37)$ & 5.17 & $-44-39-15(37)$ & 6.00 & & \\
\hline Right & & & & & & & $59-41-15(20)$ & 4.13 & & \\
\hline \multicolumn{11}{|l|}{ Occipital/cuneus } \\
\hline Left & $-54-76-10(19)$ & 5.16 & $-30-10415(18)$ & 6.50 & $-12-8834(19)$ & 5.98 & $-8-88-14(18)$ & 5.14 & $-44-96-18(18)$ & 4.42 \\
\hline Right & $38-8333(19)$ & 4.24 & $4-90-18(18)$ & 3.97 & $8-8538(19)$ & 5.02 & & & $12-1041(18)$ & 3.80 \\
\hline \multicolumn{11}{|l|}{ Cingulate } \\
\hline Left & & & & & $-4-4718(30)$ & 5.03 & $-81134(32)$ & 3.71 & $-123218(32)$ & 5.63 \\
\hline Right & 41923 (24) & 4.43 & $21037(32)$ & 4.97 & $2-468(29)$ & 5.56 & $122133(32)$ & 5.37 & $4-3535(31)$ & 3.58 \\
\hline
\end{tabular}


Table 4. Continued

\begin{tabular}{|c|c|c|c|c|c|c|c|c|c|c|}
\hline \multirow[t]{2}{*}{ Region of activation } & \multicolumn{2}{|l|}{ A1 } & \multicolumn{2}{|l|}{ A2 } & \multicolumn{2}{|l|}{$\mathbf{A 3}$} & \multicolumn{2}{|l|}{ A4 } & \multicolumn{2}{|l|}{ A5 } \\
\hline & $\mathbf{x} \mathbf{y} z(\mathbf{B A})$ & $t$ value & $\mathbf{x} \mathbf{y} z(\mathbf{B A})$ & $t$ value & $\mathbf{x} \mathbf{y} z(\mathbf{B A})$ & $t$ value & $\mathbf{x}$ y $z$ (BA) & t value & $\mathbf{x ~ y ~ z ~ ( B A ) ~}$ & $t$ value \\
\hline \multicolumn{11}{|l|}{ Parahippocampal, left } \\
\hline & $-16-14-16(28)$ & 3.53 & & & $-16-20-11(35)$ & 4.18 & $-20-1-18(34)$ & 3.92 & $-20-33-835)$ & 4.83 \\
\hline & & & & & & & $24-24-15(35)$ & 3.61 & $18-31-10(35)$ & 4.66 \\
\hline \multicolumn{11}{|l|}{ Insula } \\
\hline Left & $-28-3222(13)$ & 4.41 & 342215 (13) & 3.38 & & & $-4213-3(13)$ & 7.35 & $-4245(13)$ & 4.65 \\
\hline Right & & & & & & & & & $401211(13)$ & 5.61 \\
\hline \multicolumn{11}{|l|}{ Basal ganglia } \\
\hline Left & $-167-5$ & 4.11 & -2364 & 3.38 & $-34-214$ & 4.89 & $16-4-9$ & 3.64 & -2484 & 5.00 \\
\hline Right & & & & & & & & & $20-8-4$ & 3.51 \\
\hline \multicolumn{11}{|l|}{ Inferior frontal } \\
\hline Left & $-5138-10(47)$ & 5.07 & & & $-5726-10(47)$ & 5.48 & $-50634(9)$ & 4.07 & $-30233(47)$ & 3.34 \\
\hline Right & $6150-1(46)$ & 46.14 & & & $612423(45)$ & 5.63 & & & $59355(45)$ & 5.55 \\
\hline \multicolumn{11}{|l|}{ Middle frontal } \\
\hline Left & $40587(10)$ & 5.49 & $-613027(46)$ & 5.54 & $-50546(10)$ & 9.23 & $501132(9)$ & 4.12 & $-42-154(6)$ & 4.57 \\
\hline Right & & & & & $57440(6)$ & 6.10 & & & $302646(8)$ & 4.25 \\
\hline \multicolumn{11}{|l|}{ Superior frontal } \\
\hline Left & $2468-2(10)$ & 5.45 & $-10367(6)$ & 3.85 & $-103654(6)$ & 11.32 & $-2651(6)$ & 3.92 & $-22060(6)$ & 3.67 \\
\hline Right & & & $425123(10)$ & 4.05 & & & & & & \\
\hline \multicolumn{11}{|l|}{ Medial frontal } \\
\hline Left & $-64027(9)$ & 4.24 & $-2-2464(6)$ & 3.16 & $-63540(8)$ & 10.70 & & & $-81844(8)$ & 4.06 \\
\hline Right & & & & & $8-2666(6)$ & 6.33 & & & $6-1356(6)$ & 4.04 \\
\hline \multicolumn{11}{|l|}{ Cingulate } \\
\hline Left & $-123624(32)$ & 4.47 & $-18-3542(31)$ & 3.72 & & & & & $-14239(24)$ & 4.42 \\
\hline Right & $102823(32)$ & 4.64 & $16-4125(31)$ & 4.33 & & & & & $6443(24)$ & 4.99 \\
\hline \multicolumn{11}{|l|}{ Inferior parietal } \\
\hline Left & $-63-2427(40)$ & 3.97 & $57-4934(40)$ & 3.47 & & & $-40-5444(40)$ & 4.14 & $-53-4336(40)$ & 3.97 \\
\hline Right & $59-3024(40)$ & 4.40 & & & & & & & $34-5844(40)$ & 3.94 \\
\hline \multicolumn{11}{|l|}{ Middle temporal } \\
\hline Left & $61-35-1(21)$ & 3.94 & & & $-57-585(21)$ & 9.51 & & & $-42-5926(39)$ & 5.47 \\
\hline Right & & & & & & & & & $53-343(22)$ & 5.15 \\
\hline \multicolumn{11}{|l|}{ Superior temporal } \\
\hline Left & $-517-1(22)$ & 5.88 & $53-5516(22)$ & 3.64 & & & & & $-63-4013(22)$ & 4.71 \\
\hline Right & $63-232(22)$ & 4.79 & & & & & & & $53-259(41)$ & 5.27 \\
\hline
\end{tabular}




\begin{tabular}{|c|c|c|c|c|c|c|c|c|c|c|c|}
\hline \multirow{10}{*}{ 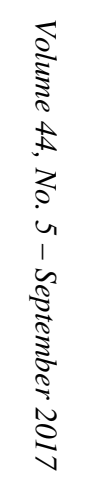 } & \multicolumn{11}{|l|}{ Cuneus/precuneus } \\
\hline & Left & $-32-8743(19)$ & 4.27 & $-30-7431(19)$ & 3.60 & $16-9525(19)$ & 5.19 & & & $-24-8148(7)$ & 5.61 \\
\hline & Right & & & $4-6948$ (7) & 3.72 & $18-1047(18)$ & 6.22 & & & $4-9527(19)$ & 3.60 \\
\hline & \multicolumn{11}{|l|}{ Fusiform/inferior temporal } \\
\hline & Left & $-61-53-16(20)$ & 4.24 & $-18-96-18(18)$ & 3.28 & & & $-2296-18(18)$ & 4.50 & $-51-45-17(37)$ & 6.27 \\
\hline & Right & & & & & & & & & $46-64-13(37)$ & 4.05 \\
\hline & \multicolumn{11}{|l|}{ Occipital } \\
\hline & Left & $-51-78-12(19)$ & 3.96 & $-34-10412(18)$ & 3.86 & $-36-9410(18)$ & 5.59 & & & $-36-7914(19)$ & 5.47 \\
\hline & Right & $53-66-5(19)$ & 4.74 & $16-91-10(17)$ & 5.46 & & & & & $12-86-11(18)$ & 4.41 \\
\hline & \multicolumn{11}{|l|}{ Thalamus } \\
\hline & Left & $-4-56$ & 4.28 & $-6-96$ & 3.87 & & & & & $-10-103$ & 4.43 \\
\hline & Right & & & & & & & & & $22-2114$ & 6.02 \\
\hline & \multicolumn{11}{|l|}{ Parahippocampal } \\
\hline & Left & $-26-34-14(36)$ & 4.13 & & & & & & & $-16-20-13(35)$ & 4.48 \\
\hline & Right & $18-385(30)$ & 3.85 & & & & & & & $34-33-10(36)$ & 5.09 \\
\hline & \multicolumn{11}{|l|}{ Insula } \\
\hline & Left & & & & & & & & & $-44-118(13)$ & 5.03 \\
\hline & \multicolumn{11}{|l|}{ Right } \\
\hline & \multicolumn{11}{|l|}{ Basal ganglia } \\
\hline & Left & -4715 & 4.30 & $-34-212$ & 3.67 & $-20-118$ & 6.46 & -1441 & 3.38 & 1285 & 3.68 \\
\hline & Right & 8917 & 4.00 & & & & & & & $14-325$ & 4.96 \\
\hline
\end{tabular}

Brodmann areas are shown in parentheses. Talairach coordinates $(\mathrm{x}, \mathrm{y}, \mathrm{z})$ are reported. 
activated in the adult stroke patients to a greater extent than the controls. Overall, activation in the adult stroke patients was more diffuse, with no consistent region of maximum activation across the group. In the picture-word matching task (Table 4, Fig. 2), adult stroke patients again showed more diffuse activation and more bilateral activation than controls. Nonetheless, strong left frontal activations were observed in all patients.

\section{Regions of Significant Activation: Childhood Stroke}

In the verb generation task (Table 5, Fig. 1), the childhood stroke patients activated many of the same regions as controls and the adult stroke patients, but activations tended to be bilateral rather than left dominant. Moreover, in contrast to the other two groups, maximum activations in the childhood stroke patients were seen in anterior cingulate (BA 24) and occipitotemporal regions, not in left frontal regions. Of note, all four childhood stroke patients activated the left and right inferior frontal gyrus to a similar extent, whereas all but one of the adult patients (A5) showed stronger left than right inferior frontal activation. None of the childhood stroke patients activated left angular gyrus and only two activated left inferior parietal cortex, though two showed right inferior parietal activation. In the picture-word matching task (Table 5, Fig. 2), maximum activations were seen in posterior regions (particularly right occipitotemporal) and, in two patients, right cingulate gyrus. Frontal activations were bilateral or predominantly right-sided. This was in contrast to the strong left frontal activations shown by controls and all of the adult stroke patients.

\section{DisCUSSION}

This is the first fMRI study to compare patterns of language lateralization following left MCA stroke in childhood and adulthood. Our study was also unique in that all participants were tested as adults, making it possible to directly compare "mature" patterns of language lateralization after childhood and adult MCA stroke in the language-dominant hemisphere. Controls showed left lateralized activation during verb generation and, to a lesser extent, during picture-word matching. Activation patterns for patients, particularly the childhood stroke group, were generally more diffuse than controls, and involved more bilateral and posterior regions. Adult stroke patients showed left lateralization for both tasks, with no statistically significant difference from controls, consistent with previous studies. ${ }^{2,3,47}$ Of note, however, the most robust activation for the adult stroke patients during verb generation was in left inferior and medial frontal regions, but outside of BA, which was the site of maximal activation in controls. Childhood stroke patients exhibited significantly weaker lateralization than the adult group for verb generation, but there was no significant group difference for picture-word matching. Maximum activations for the childhood stroke patients involved occipitotemporal cortex and anterior cingulate, in contrast to the strong left frontal activations of the adult group and controls. Our findings suggest that reorganization of language function may be more likely to involve bilateral recruitment following left MCA stroke in childhood than in adulthood. In our small sample, bilateral reorganization was most apparent on a task that is strongly left lateralized in controls (verb generation). Moreover, more diffuse left hemisphere engagement was seen in both the stroke groups compared with controls.

\section{Age at Stroke and Language Lateralization}

Our findings of bilateral language representation following childhood stroke stand in contrast to studies of adult stroke and to studies of perinatal stroke. The general consensus in the adult stroke literature is that left hemispheric reorganization (particularly involving perilesional regions) is associated with better long-term language outcome than right hemisphere dominance or bilateral representation. ${ }^{2,3,7}$ Conversely, following perinatal left MCA stroke, right hemisphere language dominance appears to be very common, even in patients with good outcome. ${ }^{8-10,48}$ This right hemisphere language shift can occur even when perinatal stroke damage is restricted to the left periventricular region ${ }^{12,13}$; however, there is some evidence that, when individual differences are examined, left inferior frontal involvement in language tasks is still advantageous in this population. ${ }^{26}$ Our study suggests that left MCA stroke during childhood may be associated with bilateral language representationessentially, a middle-ground between the adult and perinatal groups. This finding is consistent with evidence that language lateralization beings early in life ${ }^{49}$ and increases throughout childhood. ${ }^{36}$ It is also consistent with studies showing equal likelihood of language deficits in children with a history of right or left perinatal brain injury, ${ }^{28,29,50-53}$ but a strong predominance of language disorders following left hemisphere injury in older children. ${ }^{29,31,54,55}$ Thus, with increasing lateralization, the likelihood of recruiting prepotent right hemisphere regions for language following left hemisphere injury appears to quickly decrease.

However, there are some other important differences between our study of childhood stroke and those of perinatal stroke that may contribute to the different lateralization patterns. Tillema et $\mathrm{al}^{10}$ and Heller et $\mathrm{al}^{8}$ included children with active seizure disorders, whereas we excluded participants with epilepsy or other neurological comorbidities. The chronic impact of seizures on the brain may have altered lateralization patterns differently than static unilateral lesions alone. Moreover, several studies of perinatal stroke have either included children with very low IQs and/or poor language outcome $^{10}$ or they have not included any measures of language outcome. , $9,12,13$ In our study, all participants had average IQs and good language outcome documented by standardized testing; thus, differences between participants with good and poor language outcome may also have contributed to discrepancies between our findings and others. The childhood stroke group reported by Ilves and colleagues ${ }^{14}$ exhibited LIs that were more somewhat more left hemisphere dominant than ours, but lesion location information was not presented for these children, so it is difficult to compare findings directly. Finally, some studies of perinatal stroke have examined participants while they are still children, ${ }^{9,10,14,26}$ whereas our participants were young adults at the time of the study. Because of the potential differences between lateralization patterns in the developing brain versus the mature brain, it is difficult to directly compare our studies.

\section{The Impact of Cortical Versus Subcortical Lesions on Lateralization Patterns}

With respect to the lateralization differences between the adult and childhood stroke groups in our study, it is important to consider that four of the five patients in the childhood group had lesions restricted to subcortical regions with perisylvian sparing, yet all showed significant alterations in language representation. In contrast, all of the adult participants sustained lesions to 
Table 5: Areas activated by childhood stroke patients during verb generation and picture-word matching

\begin{tabular}{|c|c|c|c|c|c|c|c|c|}
\hline \multirow[b]{2}{*}{ Region of activation } & \multicolumn{2}{|l|}{ C1 } & \multicolumn{2}{|l|}{$\mathrm{C2}$} & \multicolumn{2}{|l|}{$\mathbf{C 3}$} & \multicolumn{2}{|l|}{$\mathrm{C4}$} \\
\hline & $\mathbf{x} \mathbf{y} \mathbf{z}(\mathbf{B A})$ & t value & $\mathbf{x}$ y z $(\mathbf{B A})$ & t value & $\mathbf{x} \mathbf{y} z(\mathbf{B A})$ & t value & $\mathbf{x} \mathbf{y} \mathbf{z}(\mathbf{B A})$ & t value \\
\hline \multicolumn{9}{|l|}{ Verb generation } \\
\hline \multicolumn{9}{|l|}{ Inferior frontal } \\
\hline Left & $-53357(45)$ & 2.79 & $-50355(45)$ & 3.60 & $\begin{array}{llll}-40 & 15 & -9(47)\end{array}$ & 7.79 & $-4623-11(47)$ & 4.44 \\
\hline Right & $572214(45)$ & 2.44 & 53248 (45) & 3.59 & 484311 (47) & 8.83 & $3430-9(47)$ & 5.48 \\
\hline \multicolumn{9}{|l|}{ Middle frontal } \\
\hline Left & $-30150(6)$ & 2.24 & 263027 (9) & 3.57 & $-423427(9)$ & 11.20 & $-3050-9(11)$ & 4.82 \\
\hline Right & 402942 (8) & 2.16 & & & $444422(10)$ & 6.04 & & \\
\hline \multicolumn{9}{|l|}{ Superior frontal } \\
\hline Left & $381848(8)$ & 2.44 & $-25725(9)$ & 4.78 & $-145725(9)$ & 6.12 & $-3461-15(11)$ & 4.61 \\
\hline Right & & & $145228(9)$ & 5.39 & $442046(8)$ & 6.67 & $2661-12(10)$ & 3.60 \\
\hline \multicolumn{9}{|l|}{ Medial frontal } \\
\hline Left & $-14-2257(6)$ & 2.80 & $-16-1553(6)$ & 5.28 & & & $-2048-9(10)$ & 4.00 \\
\hline Right & & & $10649(6)$ & 4.45 & & & $4846(32)$ & 5.14 \\
\hline \multicolumn{9}{|l|}{ Inferior parietal } \\
\hline Left & & & $36-4643$ & 3.16 & $-53-3527(40)$ & 7.83 & $-57-4328(40)$ & 3.56 \\
\hline Right & & & $(40)$ & & $50-3648(40)$ & 5.67 & & \\
\hline \multicolumn{9}{|l|}{ Angular gyrus } \\
\hline \multicolumn{9}{|l|}{ Left } \\
\hline \multicolumn{9}{|l|}{ Right } \\
\hline \multicolumn{9}{|l|}{ Middle temporal } \\
\hline Left & & & $44-7516(39)$ & 5.69 & $-368-36(38)$ & 6.59 & $-53-18-11(21)$ & 3.46 \\
\hline Right & & & & & $53-332(22)$ & 9.89 & $401-32(21)$ & 4.02 \\
\hline Superior temporal & 6 & & & & & & & \\
\hline Left & $1-299(42)$ & 4.03 & $-51-5312(39)$ & 3.97 & $-55-309(42)$ & 9.89 & $51122(22)$ & 5.85 \\
\hline Right & $-4014-36(38)$ & 1.75 & $4422-27(38)$ & 4.37 & $57-254(22)$ & 5.67 & & \\
\hline \multicolumn{9}{|c|}{ Fusiform/Inferior temporal } \\
\hline Left & $24-68-7(19)$ & 4.14 & $-38-43-16(37)$ & 3.42 & $-32-37-15(20)$ & 9.26 & $48-43-14(37)$ & 4.80 \\
\hline Right & & & $55-42-22(20)$ & 4.33 & & & & \\
\hline \multicolumn{9}{|l|}{ Occipital/cuneus } \\
\hline Left & $-1-856(17)$ & 2.88 & $12-56-1(19)$ & 7.37 & $-6-92-15(18)$ & 11.74 & $-2-82-10(18)$ & 7.40 \\
\hline Right & $4-91-10(18)$ & 4.64 & & & $14-83-8(18)$ & 5.48 & $26-929(18)$ & 4.13 \\
\hline \multicolumn{9}{|l|}{ Cingulate } \\
\hline Left & $-16-1434(24)$ & 1.68 & $-8-348(24)$ & 3.67 & $21928(24)$ & 5.36 & $-2926(24)$ & 4.14 \\
\hline Right & $18-1236(24)$ & 3.51 & $6-1-7(25)$ & 4.92 & & & $42223(24)$ & 4.12 \\
\hline \multicolumn{9}{|l|}{ Parahippocampal } \\
\hline Left & $-32-41-9(37)$ & 4.49 & $-24-41-5(36)$ & 3.94 & $38-28-16(36)$ & 8.36 & $32-544(30)$ & 3.24 \\
\hline Right & & & $24-37-1(27)$ & 3.26 & & & & \\
\hline Insula & & & & & & & & \\
\hline Left & & & & & & & & \\
\hline Right & & & & & & & & \\
\hline Basal ganglia & & & & & & & & \\
\hline Left & & & $-28-1514$ & 3.39 & & & 22102 & 3.50 \\
\hline Right & & & 202415 & 3.64 & & & & \\
\hline Inferior frontal & & & & & & & & \\
\hline Left & $5523-7(47)$ & 4.69 & $2011-16(47)$ & 3.69 & $-48 \quad 17-2(47)$ & 3.10 & $4633-3(47)$ & 7.12 \\
\hline Right & & & & & $484311(46)$ & 4.18 & & \\
\hline Middle frontal & & & & & & & & \\
\hline Left & $46932(9)$ & 4.10 & $-306010(10)$ & 3.53 & $-303832(9)$ & 3.74 & $-4844-6(47)$ & 4.62 \\
\hline Right & & & $382326(9)$ & 3.23 & 502328 (46) & 3.36 & $4842-10(11)$ & 3.65 \\
\hline
\end{tabular}


Table 5. Continued

\begin{tabular}{|c|c|c|c|c|c|c|c|c|}
\hline \multirow[b]{2}{*}{ Region of activation } & \multicolumn{2}{|l|}{ C1 } & \multicolumn{2}{|l|}{ C2 } & \multicolumn{2}{|l|}{$\mathbf{C 3}$} & \multicolumn{2}{|l|}{$\mathrm{C} 4$} \\
\hline & $\mathbf{x} \mathbf{y} z$ (BA) & $t$ value & $\mathbf{x} \mathbf{y} z$ (BA) & t value & $\mathbf{x} \mathbf{y} \mathbf{z}(\mathbf{B A})$ & t value & $\mathbf{x} \mathbf{y} z(\mathbf{B A})$ & t value \\
\hline \multicolumn{9}{|l|}{ Superior frontal } \\
\hline Left & $12745(10)$ & 5.14 & $-63145(8)$ & 4.03 & $101054(6)$ & 5.18 & $105229(9)$ & 3.37 \\
\hline Right & & & $2469-5(10)$ & 3.26 & & & & \\
\hline \multicolumn{9}{|l|}{ Medial frontal } \\
\hline Left & $-64829(9)$ & 3.36 & $-4-157(6)$ & 4.24 & $81643(6)$ & 4.71 & $-84829(9)$ & 5.42 \\
\hline Right & & & $8-553(6)$ & 4.24 & & & $164717(10)$ & 4.02 \\
\hline \multicolumn{9}{|l|}{ Cingulate } \\
\hline Left & $-639-1(32)$ & 3.31 & $-21643(32)$ & 4.25 & $21928(24)$ & 3.20 & $-24-3142(31)$ & 4.48 \\
\hline Right & $8-5316(30)$ & 6.17 & $82526(24)$ & 5.22 & & & $41037(32)$ & 3.64 \\
\hline \multicolumn{9}{|l|}{ Inferior parietal } \\
\hline Left & & & $-61-2625(40)$ & 3.18 & $-55-3330(40)$ & 3.09 & & \\
\hline \multicolumn{9}{|l|}{ Right } \\
\hline \multicolumn{9}{|l|}{ Middle temporal } \\
\hline Left & $-631-12(21)$ & 4.59 & $-51-39-7(20)$ & 3.63 & $-368-36(38)$ & 3.39 & $-63-426(22)$ & 3.19 \\
\hline Right & $40-6520(39)$ & 6.78 & $61-52-3(37)$ & 4.74 & $53-332(20)$ & 4.53 & & \\
\hline \multicolumn{9}{|l|}{ Superior temporal } \\
\hline Left & $-53-5317(22)$ & 4.84 & $-4620-24(38)$ & 4.70 & $-51-255(41)$ & 4.65 & $321235(38)$ & 3.71 \\
\hline Right & $46-5516(22)$ & 6.12 & $4614-21(38)$ & 4.51 & $65-114(22)$ & 3.76 & & \\
\hline \multicolumn{9}{|l|}{ Cuneus/precuneus } \\
\hline Left & & & $-12-5636(31)$ & 3.76 & $-88-8426(19)$ & 3.80 & & \\
\hline Right & & & $4-6634(7)$ & 5.30 & $20-951(17)$ & 3.60 & & \\
\hline \multicolumn{9}{|c|}{ Fusiform/inferior temporal } \\
\hline Left & $-44-72-10(19)$ & 3.74 & $-59-22-25(20)$ & 3.36 & $53-11-29(20)$ & 3.28 & $-40-30-18(20)$ & 3.29 \\
\hline Right & $22-90-18(18)$ & 8.87 & $55-47-9(20)$ & 3.95 & $46-5-35(20)$ & 3.41 & $53-64-12(37)$ & 3.45 \\
\hline \multicolumn{9}{|l|}{ Occipital } \\
\hline Left & $28-87-4(18)$ & 7.81 & & & $-18-896(17)$ & 3.22 & & \\
\hline Right & $-18-89-3(17)$ & 7.53 & & & $8-892(17)$ & 3.61 & & \\
\hline \multicolumn{9}{|l|}{ Thalamus } \\
\hline Left & $8-2419$ & 3.15 & $-6-2421$ & 3.40 & & & & \\
\hline Right & & & $12-2221$ & 3.67 & & & & \\
\hline \multicolumn{9}{|l|}{ Parahippocampal } \\
\hline \multicolumn{9}{|l|}{ Left } \\
\hline \multicolumn{9}{|l|}{ Right } \\
\hline \multicolumn{9}{|l|}{ Insula } \\
\hline Left & $44-4414(13)$ & 4.55 & $32-1026(13)$ & 4.58 & $-44-232(13)$ & 4.68 & & \\
\hline Right & & & & & $50-3220(13)$ & 4.36 & & \\
\hline \multicolumn{9}{|l|}{ Basal ganglia } \\
\hline Left & -14241 & 3.19 & 101619 & 3.25 & -1281 & 3.14 & & \\
\hline Right & $28-4216$ & 3.64 & & & $282-7$ & 3.56 & & \\
\hline
\end{tabular}

Brodmann areas are shown in parentheses. Talairach coordinates $(\mathrm{x}, \mathrm{y}, \mathrm{z})$ are reported.

canonical language areas in the left frontoparietal cortex, which one might expect to result in more pronounced alterations of language representation.

There are few data on aphasia following subcortical lesions in children, ${ }^{56-60}$ but the literature examining subcortical aphasia following adult stroke is quite extensive. ${ }^{61-65}$ The role of the left thalamus in language function (particularly word finding) has been firmly established, ${ }^{66-69}$ but there is considerable debate about the role of the basal ganglia. Aphasic deficits following left basal ganglia stroke in adults have been widely reported, but there does not appear to be a particular aphasic syndrome associated with such lesions. ${ }^{60,65,66}$ Several theories regarding the neurological mechanism of basal ganglia aphasia have attempted to explain symptom variability across patients. Perhaps the most compelling theory is that the underlying large vessel stenosis that causes the basal ganglia infarct also results in cortical hypoperfusion, which, in turn, disrupts language function. Hillis and colleagues found cortical hypoperfusion in patients with basal 
ganglia infarcts was strongly associated with aphasia and that symptoms resolved with perfusion normalization. ${ }^{47,70,71}$ Moreover, the regional location of the hypoperfusion predicted the specific aphasic deficits that were manifest in each patient. ${ }^{72}$ Also, in a sample of children and young adults with basal ganglia stroke, Rowan et $\mathrm{al}^{73}$ found that language deficits were associated with subtle abnormalities in cortical language areas, detected only through voxel-based morphometry. Extrapolating these findings to the context of our study, one could speculate that large-vessel stenosis and basal ganglia infarction in childhood could disrupt cortical perfusion within the dominant hemisphere, thereby impacting the way in which cortical-subcortical language networks develop. Furthermore, because the diagnosis and treatment of pediatric stroke is often delayed, ${ }^{74}$ children may be at increased risk for long periods of cortical hypoperfusion. Of note, all of the pediatric participants included in our study had documented stenosis of the left internal carotid artery and/or left MCA at the time of the stroke, making this a plausible explanation for our findings. We did not examine resting cortical perfusion in our participants, but this would be an interesting element to incorporate into future studies.

\section{Task Differences and Lateralization Patterns}

Another important finding to highlight from our study is that LIs and activation patterns varied considerably between the verb generation and picture-word matching tasks. This was true for controls and both patient groups. Verb generation is one of the most commonly used language task in neuroimaging studies and has been associated with strongly lateralized activation patterns in control participants ${ }^{34,36}$ and clinical populations. ${ }^{6,35}$ Thus, we reasoned that this task would be particularly useful for contrasting intra- and inter-hemispheric reorganization of language function following left MCA stroke in childhood and adulthood. As hypothesized, the verb generation task was most informative in distinguishing between the activation patterns of the three groups, with controls showing stronger left lateralization than patients and the adult stroke group showing stronger left lateralization than the childhood stroke group. In comparison, the picture-word matching task was not as good at distinguishing among the three groups in our study, although the generation trend toward stronger left lateralization in control versus patients and in adult stroke participants versus childhood stroke participants was still observed. Other studies using similar tasks involving picture-word matching or overt picture naming indicate that the associated patterns of brain activation are less consistent-and less strongly lateralized-than those during verb generation ${ }^{6,34}$; thus, it is perhaps not surprising that our picture-word matching task was less powerful than the verb generation task for investigating our hypotheses. Ours is not the first study to document task differences in lateralization, ${ }^{2,37}$ and it is widely recognized that task differences across studies contribute significantly to inconsistencies in the literature. ${ }^{6,35,38}$ As a result, it is critical to consider task demands when interpreting patterns of language lateralization, both in normal participants and clinical populations.

\section{Future Directions}

Our study is limited by small sample sizes and differences in lesion location across the adult and childhood stroke groups. We are currently recruiting participants with cortical left MCA stroke in childhood to address this confound. A cortical childhood stroke group would be an important contrast to both the adult stroke and subcortical childhood groups included in the present study because it would permit more definitive conclusions about the impact of lesion location versus age at lesion on reorganization patterns. Moreover, it would be informative to follow children with left MCA stroke over time with repeated fMRI sessions to examine how lateralization changes with recovery and development. Another limitation of our study is the significant difference in age at study between the pediatric and adult stroke groups. As a result, factors related to neural development (such as brain atrophy related to aging and continued brain maturation in young adults) may have affected our findings. The pediatric and adult stroke groups also differ in number of years since stroke, with the adult group having fewer years to develop compensatory strategies and brain networks. This may have also affected our findings, although based on previous research, ${ }^{2,3,6}$ one might expect that a shorter length of time since stroke would make it more likely that the adult stroke participants would show such strong left lateralization. Longitudinal studies that follow childhood stroke survivors throughout the lifespan are needed to address important questions related to the impact of an early lesion on the aging process. Stroke is the ideal population in which to study structurefunction relationships and age-related changes in plasticity because the lesions are static, focal, and can occur at any age. However, because of the relatively low incidence of pediatric stroke, ${ }^{75}$ opportunities to directly contrast childhood and adult stroke are rare. Multicenter studies may allow for larger sample sizes, more tightly matched subject groups, and statistical analysis of individual differences.

\section{DisClosures}

The authors have nothing to disclose.

\section{REFERENCES}

1. Woods BT, Teuber HL. Early onset of complementary specialization of cerebral hemispheres in man. Trans Am Neurol Assoc. 1973;98:113-7.

2. Calvert GA, Brammer MJ, Morris RG, Williams SC, King N, Matthews PM. Using fMRI to study recovery from acquired dysphasia. Brain Lang. 2000;71:391-9.

3. Cao Y, Vikingstad EM, George KP, Johnson a F, Welch KM. Cortical language activation in stroke patients recovering from aphasia with functional MRI. Stroke. 1999;30:2331-40.

4. Heiss WD, Karbe H, Weber-Luxenburger G, et al. Speech-induced cerebral metabolic activation reflects recovery from aphasia. J Neurol Sci. 1997;145:213-7.

5. Karbe H, Thiel A, Weber-Luxenburger G, Herholz K, Kessler J, Heiss WD. Brain plasticity in poststroke aphasia: what is the contribution of the right hemisphere? Brain Lang. 1998;64:215-30.

6. Price CJ, Crinion J. The latest on functional imaging studies of aphasic stroke. Curr Opin Neurol. 2005;18:429-34.

7. Xu XJ, Zhang MM, Shang DS, Wang QD, Luo BY, Weng XC. Cortical language activation in aphasia: a functional MRI study. Chin Med J (Engl). 2004;117:1011-6.

8. Heller SL, Heier LA, Watts R, et al. Evidence of cerebral reorganization following perinatal stroke demonstrated with fMRI and DTI tractography. Clin Imaging. 2005;29:283-7.

9. Jacola LM, Schapiro MB, Schmithorst VJ, et al. Functional magnetic resonance imaging reveals atypical language organization in children following perinatal left middle cerebral artery stroke. Neuropediatrics. 2006;37:46-52. 
10. Tillema JM, Byars AW, Jacola LM, et al. Cortical reorganization of language functioning following perinatal left MCA stroke. Brain Lang. 2007;105:99-111.

11. Vikingstad EM, Cao Y, Thomas AJ, Johnson AF, Malik GM, Welch KMA. Language hemispheric dominance in patients with congenital lesions of eloquent. Neurosurgery. 2000;47:562-70.

12. Staudt M, Grodd W, Niemann G, Wildgruber D, Erb M, KrägelohMann I. Early left periventricular brain lesions induce right hemispheric organization of speech. Neurology. 2001;57:122-5.

13. Staudt M, Lidzba K, Grodd W, Wildgruber D, Erb M, KrägelohMann I. Right-hemispheric organization of language following early left-sided brain lesions: functional MRI topography. Neuroimage. 2002;16:954-67.

14. Ilves P, Tomberg T, Kepler J, et al. Different plasticity patterns of language function in children with perinatal and childhood stroke. J Child Neurol. 2014;29:756-64.

15. Liégeois F, Connelly A, Cross JH, et al. Language reorganization in children with early-onset lesions of the left hemisphere: an fMRI study. Brain. 2004;127:1229-36.

16. Muller RA, Behen ME, Rothermel RD, Muzik O, Chakraborty PK, Chugani HT. Brain organization for language in children, adolescents, and adults with left hemisphere lesion: a PET study. Prog Neuropsychopharmacol Biol Psychiatry. 1999;23: 657-68.

17. Muller RA, Rothermel RD, Behen ME, Muzik O, Chakraborty PK, Chugani HT. Language organization in patients with early and late left-hemisphere lesion: a PET study. Neuropsychologia. 1999;37:545-57.

18. Müller RA, Rothermel RD, Behen ME, et al. Brain organization of language after early unilateral lesion: a PET study. Brain Lang. 1998:62:422-51.

19. Hurford JR. The evolution of the critical period for language acquisition. Cognition. 1991;40:159-201.

20. Redcay E, Haist F, Courchesne E. Functional neuroimaging of speech perception during a pivotal period in language acquisition. Dev Sci. 2008;11:237-52.

21. Vargha-Khadem F, Isaacs EB, Papaleloudi H, Polkey CE, Wilson J. Development of language in six hemispherectomized patients. Brain. 1991;114:473-95.

22. Holland SK, Plante E, Weber Byars a, Strawsburg RH, Schmithorst VJ, Ball WS. Normal fMRI brain activation patterns in children performing a verb generation task. Neuroimage. 2001; 14:837-43.

23. Anderson DP, Harvey AS, Saling MM, et al. fMRI lateralization of expressive language in children with cerebral lesions. Epilepsia. 2006;47:998-1008

24. Duchowny M, Jayakar P, Harvey AS, et al. Language cortex representation: effects of developmental versus acquired pathology. Ann Neurol. 1996;40:31-8.

25. DeVos KJ, Wyllie E, Geckler C, Kotagal P, Comair Y. Language dominance in patients with early childhood tumors near left hemisphere language areas. Neurology. 1995;45:349-56.

26. Raja Beharelle A, Dick AS, Josse G, et al. Left hemisphere regions are critical for language in the face of early left focal brain injury. Brain. 2010;133:1707-16.

27. Dennis M. Developmental plasticity in children. J Commun Disord. 2000;33:321-32.

28. Nass R. Language development in children with congenital strokes Vol. 4 Semin Pediatr Neurol. 1997:109-16.

29. Dennis M. Discourse in children with neurodevelopmental disorder, early focal brain injury, or childhood acquired brain injury. Brain Lang. 1998;307:305-7.

30. Eisele JA, Lust B, Aram DM. Presupposition and implication of truth: linguistic deficits following early brain lesions. Brain Lang. 1998;61:376-94.

31. Woods BT, Carey S. Language deficits after apparent clinical recovery from childhood aphasia. Ann Neurol. 1979;6:405-9.

32. Kolb B. Brain development, plasticity, and behavior. Am Psychol. 1989;44:1203-12.

33. Vargha-Khadem F. Generalized versus selective cognitive impairments resulting from brain damage sustained in childhood. Epilepsia. 2001;42(Suppl 1):37-40
34. Pizzamiglio L, Galati G, Committeri G. The contribution of functional neuroimaging to recovery after brain damage: a review. Cortex. 2001;3:11-31.

35. Rijntjes M, Weiller C. Recovery of motor and language abilities after stroke: the contribution of functional imaging. Prog Neurobiol. 2002;66:109-22.

36. Schlaggar BL, Brown TT, Lugar HM, Visscher KM, Miezin FM, Petersen SE. Functional neuroanatomical differences between adults and school-age children in the processing of single words. Science. 2002;296:1476-9.

37. Barnett A, Marty-Dugas J, McAndrews MP. Advantages of sentencelevel fMRI language tasks in presurgical language mapping for temporal lobe epilepsy. Epilepsy Behav. 2014;32:114-20.

38. Gaillard WD, Balsamo L, Xu B, et al. fMRI language task panel improves determination of language dominance. Neurology. 2004;63:1403-8

39. Müller R a, Rothermel RD, Behen ME, Muzik O, Mangner TJ, Chugani HT. Differential patterns of language and motor reorganization following early left hemisphere lesion: a PET study. Arch Neurol. 1998;55:1113-9.

40. Cardenas JR, Rho JM, Kirton A. Pediatric stroke. Child's Nerv Syst. 2011;27:1375-90.

41. Carvalho KS, Garg BP. Arterial strokes in children Vol. 20 Neurol Clin. 2002:1079-100.

42. Askalan R, Laughlin S, Mayank S, et al. Chickenpox and stroke in childhood: a study of frequency and causation. Stroke. 2001;32:1257-62.

43. DeVeber G, Kirton A, Booth F, et al. Epidemiology and outcomes of arterial ischemic stroke in children: the Canadian Pediatric Ischemic Stroke Registry. Pediatr Neurol. 2017;69:58-70.

44. Wellcome Trust Centre for Neuroimaging. Statistical parametric mapping. University College London, 2002. Available at: http:// www.fil.ion.ucl.ac.uk/spm/doc.

45. Catani M, Jones DK, Ffytche DH. Perisylvian language networks of the human brain. Ann Neurol. 2005;57:8-16.

46. Rutten GJM, Ramsey NF, van Rijen PC, van Veelen CWM. Reproducibility of fMRI-determined language lateralization in individual subjects. Brain Lang. 2002;80:421-37.

47. Hillis AE, Wityk RJ, Tuffiash E, et al. Hypoperfusion of Wernicke's area predicts severity of semantic deficit in acute stroke. Ann Neurol. 2001;50:561-6.

48. Szaflarski JP, Allendorfer JB, Byars AW, et al. Age at stroke determines post-stroke language lateralization. Restor Neurol Neurosci. 2014;32:733-42.

49. Dehaene-Lambertz G, Dehaene S, Hertz-Pannier L. Functional neuroimaging of speech perception in infants. Science. 2002;298:2013-5.

50. Vargha-Khadem F, O'Gorman AM, Watters GV. Aphasia and handedness in relation to hemispheric side, age at injury and severity of cerebral lesion during childhood. Brain. 1985;108:677-96.

51. Thal DJ, Marchman V, Stiles J, et al. Early lexical development in children with focal brain injury. Brain Lang. 1991;40:491-527.

52. Bates EA. Explaining and interpreting deficits in language development across clinical groups: where do we go from here? Brain Lang. 2004;88:248-53.

53. Trauner DA, Eshagh K, Ballantyne AO, Bates E. Early language development after peri-natal stroke. Brain Lang. 2013;127:399-403.

54. Nass RD, Trauner D. Social and affective impairments are important recovery after acquired stroke in childhood. CNS Spectr. 2004:9:420-34.

55. Van Hout A. Acquired aphasia in children. Semin Pediatr Neurol. 1997:4:102-8.

56. Aram DM, Rose DF, Rekate HL, Whitaker HA. Acquired capsular/ striatal aphasia in childhood. Arch Neurol. 1983;40(10):614-7.

57. Cranberg LD, Filley CM, Hart EJ, Alexander MP. Acquired aphasia in childhood: clinical and CT investigations. Neurology. 1987;37:1165-72.

58. Ferro JM, Martins IP, Pinto F, Castro-Caldas A. Aphasia following right striato-insular infarction in a left-handed child: a clinicoradiological study. Develop Med Child Neurol. 1982;24:173-8.

59. van Lieshout P, Renier W, Eling P, de Bot K, Slis I. Bilingual language processing after a lesion in the left thalamic and 
temporal regions. A case report with early childhood onset. Brain Lang. 1990;38:173-94.

60. Gout A, Seibel N, Rouvière C, et al. Aphasia owing to subcortical brain infarcts in childhood. J Child Neurol. 2005;20:1003-8.

61. Perani D, Vallar G, Cappa S, Messa C, Fazio F. Aphasia and neglect after subcortical stroke: a clinical/cerebral PERFUSION correlation study. Brain. 1987;110:1211-29.

62. Perani D, Di Piero V, Lucignani G, et al. Remote effects of subcortical cerebrovascular lesions: a SPECT cerebral perfusion study. J Cerebral Blood Flow Metab. 1988;8:560-7.

63. Vallar G, Perani D, Cappa SF, Messa C, Lenzi GL, Fazio F. Recovery from aphasia and neglect after subcortical stroke: neuropsychological and cerebral perfusion study. J Neurol Neurosurg Psychiatry. 1988;51:1269-76.

64. Damasio AR, Damasio H, Rizzo M, Varney N, Gersch F. Aphasia with nonhemorrhagic lesions in the basal ganglia and internal capsule. Arch Neurol. 1982;39:15-20.

65. Nadeau SE, Crosson B. Subcortical aphasia. Brain Lang. 1997;58:355-402; discussion 418-23.

66. Crosson B. Subcortical functions in language: a working model. Brain Lang. 1985;25:257-92.

67. Ojemann GA. Functional mapping of cortical language areas in adults. Intraoperative approaches. Adv Neurol. 1993;63:155-63.
68. Cappa SF, Vignolo LA. "Transcortical” features of aphasia following left thalamic hemorrhage. Cortex. 1979;15:121-30.

69. Kumar R, Masih a K, Pardo J. Global aphasia due to thalamic hemorrhage: a case report and review of the literature. Arch Phys Med Rehabil. 1996;77:1312-5.

70. Hillis AE, Kane A, Tuffiash E, et al. Reperfusion of specific brain regions by raising blood pressure restores selective language functions in subacute stroke. Brain Lang. 2001;79: 495-510.

71. Hillis AE, Barker PB, Beauchamp NJ, Winters BD, Mirski M, Wityk RJ. Restoring blood pressure reperfused Wernicke's area and improved language. Neurology. 2001;56:670-2.

72. Hillis AE, Barker PB, Wityk RJ, et al. Variability in subcortical aphasia is due to variable sites of cortical hypoperfusion. Brain Lang. 2004;89:524-30.

73. Rowan A, Vargha-Khadem F, Calamante F, et al. Cortical abnormalities and language function in young patients with basal ganglia stroke. Neuroimage. 2007;36:431-40.

74. Rafay MF, Pontigon AM, Chiang J, et al. Delay to diagnosis in acute pediatric arterial ischemic stroke. Stroke.. 2009;40:58-64.

75. deVeber G, Canadian Paediatric Ischemic Stroke Study Group. Canadian Paediatric Ischemic Stroke Registry: analysis of children with arterial ischemic stroke. Ann Neurol. 2000;48:514. 